\title{
Secondary Metabolites: Involvement in Abiotic Stress Tolerance of Cotton
}

\author{
Aglika Edreva* \\ Institute of Plant Physiology and Genetics, Bulgarian Academy of Sciences, Bulgaria
}

Submission: January 31, 2018; Published: February 19, 2018

*Corresponding author: Aglika Edreva, Institute of Plant Physiology and Genetics, Bulgarian Academy of Sciences, 1113 Sofia, Bulgaria, Email: edreva5@yahoo.com

\section{Mini Review}

The term "secondary" introduced by A. Kossel in 1891 implies that while primary metabolites are present in every living cell capable of dividing, the secondary metabolites are present only "incidentally" and are not of paramount significance for plant life. They play a major role in the adaptation of the plant to the constantly changing environment and in overcoming stress constraints. This flows from the large complexity of chemical types and interactions underlying various functions: structure stabilizing determined by polymerization and condensation of phenols and quinones, or by electrostatic interactions of positively charged molecules with negatively charged loci in cell components; photoprotective related to absorbance of visible light and UV-radiation due to the presence of conjugated double bonds; antioxidant and antiradical governed by the availability of hydroxy, o-dihydroxy, amine, and sulfhydryl groupings, as well as by aromatic nuclei and unsaturated aliphatic chains; signal transducing.

In collaborative works with colleagues from Ege University, Izmir, Turkey (Aynur Gürel and Lale Yildiz-Aktas), Soleya Dagnon from Plovdiv University, Bulgaria, and Emilya Gesheva from the Institute of Plant Physiology and Genetics, Sofia, we confirmed the important role of secondary metabolites as a non-enzymatic tool for stress protection of plants by using two model systems for field experiments in the Aegean region of Turkey:

a. Cotton - Na-K imbalance due to leakage of $\mathrm{K}$ from the soil and plants, and over-accumulation of $\mathrm{Na}$ in the leaves reported to provoke a state of oxidative stress. Phenotypically it is expressed as reddening of upper leaves of plants. This physiological disorder is distributed in the Aegean region of Turkey (depending on soil conditions), better expressed at the beginning of September, and common for all cotton cultivars. It leads to disturbance of plant growth and development and to economic losses.In our experiments upper green leaves from plants not affected by reddening were used as controls. b. Cotton - water shortage provoking drought and a state of oxidative stress.To this purpose two irrigation regimes were applied: normal water supply (field capacity) (control), and limited water supply (1/3 field capacity) causing drought. Genotypes with contrasting drought tolerance were used: Nazilli 84, drought sensitive, and Sahin 2000, drought tolerant.

Working with the first system, we analyzed the polyphenol complex since polyphenols are one of the major classes of secondary metabolites in plants. HPLC (High Performance Liquid Chromatography) was applied. We recorded the presence of 3 groups of polyphenols: cinnamic acid derivatives (C6-C3); flavonoids (C6-C3-C6); and anthocyanins (C6-C3-C6+). In the first group we identified neochlorogenic acid (5-0-caffeoyl quinic acid) and chlorogenic acid (3-0-caffeoyl quinic acid). In the group of flavonoids isoquercitrin, rutin, quercitrin and quercetin were identified. In these two groups no quantitative and qualitative differences between green and red leaves were observed. In contrast, anthocyanins, endowed with antioxidant and antiradical properties, accumulated up to 6-7 fold in the red leaves as compared to control green leaves. Noticeably, the aglycone moiety of anthocyanins in the green leaves was identified as malvidin while the aglycone of the red leaves was determined as cyanidin. These aglycones are distinguished by the presence of an ortho-dihydroxy grouping in the B-ring of cyanidin while this grouping lacks in malvidin which is monohydroxylated. The presence of ortho-dihydroxy grouping in the molecule determines easy electron and energy transfers and hence a greater antioxidant and antiradical power. This character was demonstrated in model in vitro systems by precise physical techniques, and confirmed in experiments with mutant and transgenic plants. The fact that no changes in cinnamic acid derivatives and flavonoids occur during reddening of cotton suggests that in these specific stressful conditions the biosynthetic potential of plants is directed to accumulation of anthocyanins as a strategy to combat the oxidative stress 
constraint. Moreover, the shift from malvidin to cyanidin aglycones, i.e. the change of the hydroxylation pattern of the B-ring from mono- to o-dixydroxy substitution determines a better capacity of reddening cotton plants to counteract oxidative stress imposed by Na over-accumulation. The better antioxidant protection of the red leaves is substantiated also by the fact that the lipid peroxidation indicative of membrane damage and fragmentation was not increased as shown by thiobarbituric acid test; conserved membrane integrity in the red leaves was also demonstrated by transmission electron microscopy [1-3].

Drought is commonly defined as the absence of adequate moisture for a plant to grow normally and complete its life cycle. In conjunction with coincident high temperature and irradiance, drought poses the most important environmental constraints to plant survival, distribution and crop productivity causing important economic losses. In our experiments we investigated the polyphenol complex by means of HPLC, the antiradical activity by the DPPH assay, and the membrane state by the thiobarbituric acid test. Drought sensitive and drought tolerant cotton cultivars showed similar polyphenol patterns in which we identified the cinnamic acid derivatives neochlorogenic acid ( 5-0-caffeoyl quinic acid), chlorogenic acid (3-0-caffeoylquinic acid), and 4-0- caffeoyl quinic acid as well as the flavonoid glycosides isoquercitrin, rutin, and kaemferol3-rutinoside having quercetin (o-dihydroxy substituted B-ring) or kaempferol (monohydroxy substituted B-ring) as aglycones. Noticeably, quercetin glycosides predominate over kaempferol glycosides. In stress-free conditions the tolerant genotype Sahin 2000 is distinguished by a higher pool of polyphenols, higher antiradical activity, and better protected membranes than the sensitive Nazilli 84. This can be regarded as a greater potential of the tolerant genotype to trigger a more effective antioxidant defense when drought constraint will be imposed. Upon water shortage the components of the polyphenol complex were decreased, the antiradical capacity was also decreased, while membrane integrity suffered damage. It has to be emphasized that this response was less pronounced in the tolerant than in the sensitive genotype, i.e. despite the drought stress conditions imposed, the tolerant plants maintained a more effective defense system.The data point to the importance of the o-dihydroxy substitution of the B-ring in flavonoids such as quercetin as a rationale of a stronger antioxidant and antiradical power. Chelation of $\mathrm{Fe}$ ions by quercetin interferes with the Fenton reaction producing reactive oxygen species (ROS), and can also be of key importance in the antioxidant defense performed by secondary metabolites thus contributing to improved abiotic stress tolerance.

\section{References}

1. Edreva A, Dagnon S, Gürel A, Gesheva E, Hakerlerler H (2006) Agrochimica. L-N (1-2): 54-61.

2. Yildiz-Aktas L, Dagnon, Gurel A, Gesheva E, Edreva A (2009) J Agronomy \& Crop Science, 195, 147-253.

3. Edreva A, Velikova V, Tsonev T, Dagnon S, Gurel A, et al. (2008) Gen Appl Plant Physiology 34(1-2): 67-77.
Your next submission with Juniper Publishers will reach you the below assets

- Quality Editorial service

- Swift Peer Review

- Reprints availability

- E-prints Service

- Manuscript Podcast for convenient understanding

- Global attainment for your research

- Manuscript accessibility in different formats

( Pdf, E-pub, Full Text, Audio)

- Unceasing customer service

Track the below URL for one-step submission https://juniperpublishers.com/online-submission.php 